\title{
Water in Ionic Liquid for Electrochemical Li Cycling
}

Fuminori Mizuno ${ }^{1,2}$, Timothy S. Arthur ${ }^{1, *}$ and Kensuke Takechi ${ }^{1}$

${ }^{1}$ Toyota Research Institute of North America, 1555 Woodridge Avenue, Ann Arbor, MI 48105, USA

${ }^{2}$ Toyota Motor Corporation, Higashifuji Technical Center, 1200 Mishuku, Susono, Shizuoka 410-1193, Japan

* Corresponding author: Timothy S. Arthur (tim.arthur@toyota.com)

\section{Supporting Information}

I. Experimental

a. Electrolyte preparation

b. Electrochemical analyses

c. Chemical and physical analyses

II. Figures

a. Figure S1. $(a, c)$ O1s and (b,d) F1s XPS HRES spectra of the Li metal surface after OCV hold (thin line) and galvanic cycling (thick lines) in in dry (black) and wet (blue) PC electrolyte.

b. Figure S2. Electrochemical impedance spectroscopy of as-received PP13TFSI electrolyte (black diamond) and after addition of $1 \mathrm{vol} \% \mathrm{H}_{2} \mathrm{O}$.

c. Figure S3. DSC profile of PP13TFSI electrolyte with $0.352 \mathrm{~mol} \mathrm{~kg}^{-1}$ LiTFSI (solid line). Neat PP13TFSI is also shown as a reference (dashed line). 


\section{Experimental}

\section{Electrolyte preparation}

Battery grade N-methyl-N-propylpiperidinium bis(trifluoromethanesulfonyl)imide (PP13-TFSI) as ionic liquid was purchased from Kanto Corporation (Japan). Battery grade propylene carbonate (PC) as aprotic organic solvent and lithium bis(trifluoromethanesulfonyl)imide (LiTFSI) as electrolyte salt was purchased from Kishida Chemical Co. (Japan). LiTFSI was dried at $120{ }^{\circ} \mathrm{C}$ under vacuum overnight. Water contents of as-received solvents were confirmed by a Karl Fisher titrator (Mettler Toledo, C30S) to make sure that it was less than at least $50 \mathrm{ppm}$. Three kinds of electrolyte solutions were prepared for this study. First, $0.352 \mathrm{~mol} \mathrm{~kg}^{-1}$ of LiTFSI was mixed with the as-received solvents in a Ar-filled glove box and stirred overnight to prepare standard electrolyte solutions. Second, the obtained electrolyte solutions were exposed overnight to a dry air atmosphere where the dew point was $-20{ }^{\circ} \mathrm{C}$. Third, $1 \%$ of deionized water was added to the as-made electrolyte solution in the dry air atmosphere. In all cases, no water bubble, no precipitation and no liquid phase separation were visibly observed. Neat PP13TFSI ionic liquid was separately prepared by vacuum drying of as-received one at $120{ }^{\circ} \mathrm{C}$ overnight. Finally, water contents of all the electrolyte solutions were monitored again by Karl Fisher titration. In this study, the first, second and third electrolyte solutions were called dry, moderate and wet electrolytes, respectively.

\section{Electrochemical analyses}

A standard CR2032 coin cell made of stainless steel was assembled with two sheets of Li foils (Battery grade, FMC) and a sheet of glass microfiber separator (Whatman). Li foils were polished prior to use and sandwiched the separator. As-made electrolyte solution was just poured by dropcast in the porous separator. All the processes for coin cell assembly were done in a Ar-filled glove box. After constructing the cell, charge-discharge measurements (VMP3, Biologic) meaning Li deposition and stripping were repeatedly done under constant current density $\left( \pm 0.1 \mathrm{~mA} / \mathrm{cm}^{2}\right)$ at room temperature. The applied time for each charge and discharge was 34 minutes, which corresponds to $0.1 \mathrm{mAh}$ for Li metal transported back and forth. For the reference, all series of cells were just kept at open circuit condition 
without any electrochemical schedule. Electrochemical impedance spectroscopy was also carried out at open circuit condition before and after measurements.

\section{Chemical and physical analyses}

X-ray photoelectron spectroscopy (XPS) was performed to see chemical information on a Li metal surface. X-ray photoelectron spectroscopy was collected using a Phi 5600 and analyzed using the Multipack software. Spectra were aligned to the C1s signal at $284.7 \mathrm{eV}$. To keep the surface condition unchanged, the Li metal surface was analyzed under as-tested conditions. After electrochemical testing, the electrolyte was removed physically by gentle application of a clean wipe, without immersing the Li metal in a rinsing solvent, and dried in vacuum for $12 \mathrm{~h}$. All the processes were performed in an Ar-filled glove box. Then, the sample was loaded into a XPS chamber in a sealed transfer vessel. Finally, the Li metal was analyzed in detail after $\mathrm{Ar}^{+}$sputtering for 2 minutes.

Differential scanning calorimetry (DSC) was conducted to measure the thermal properties of asprepared electrolyte solutions. A droplet of solution was poured in an Al pan and sealed well in a Arfilled glove box. The sample was loaded in a DSC machine (TA instruments, Q100) and then measured with purging $\mathrm{N}_{2}$ gas. The scan rate was $2{ }^{\circ} \mathrm{C} / \mathrm{min}$ with cooling first and the temperature range was $-90{ }^{\circ} \mathrm{C}$ to $40^{\circ} \mathrm{C}$. 
II. Figures

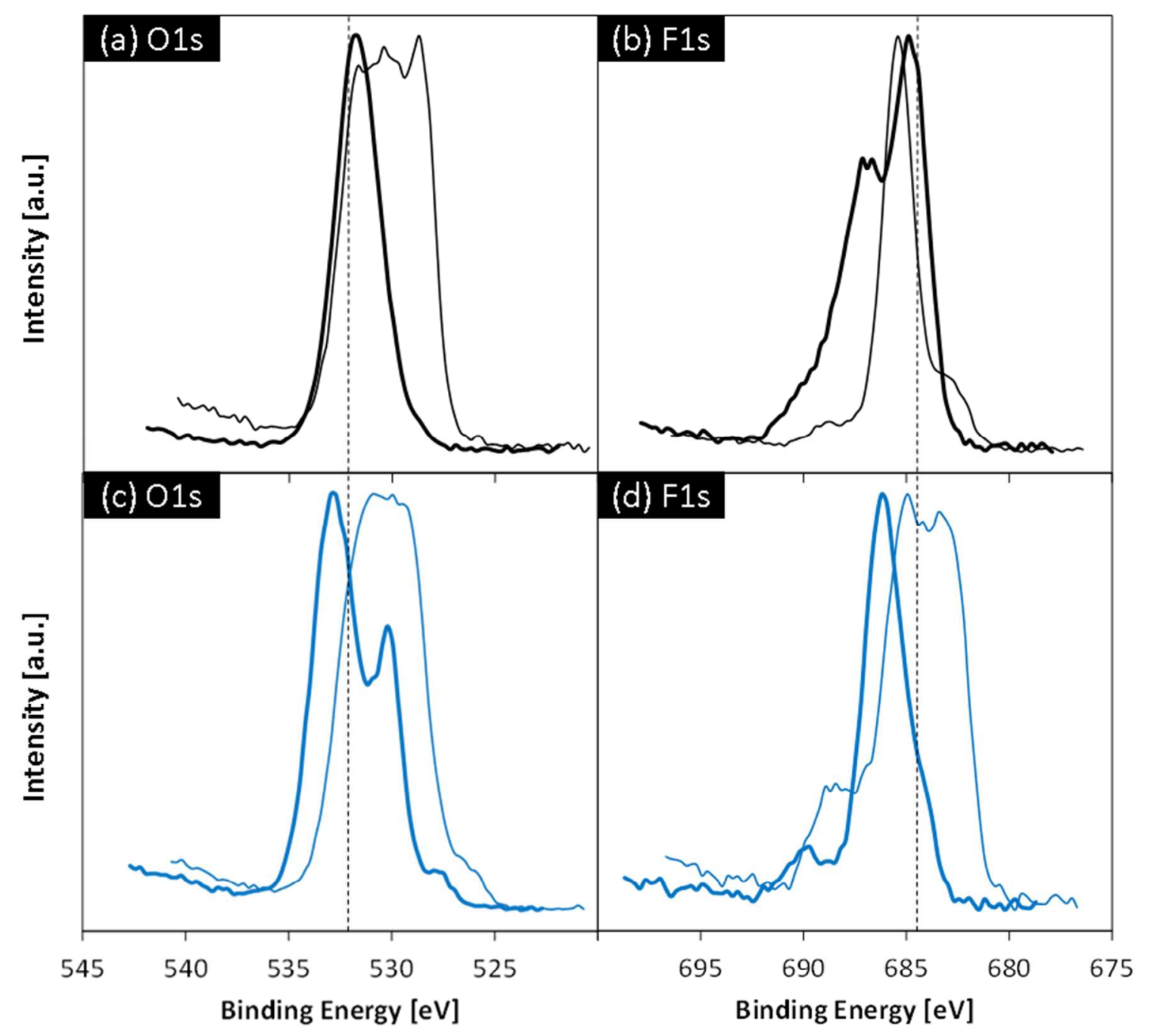

Figure S1. 


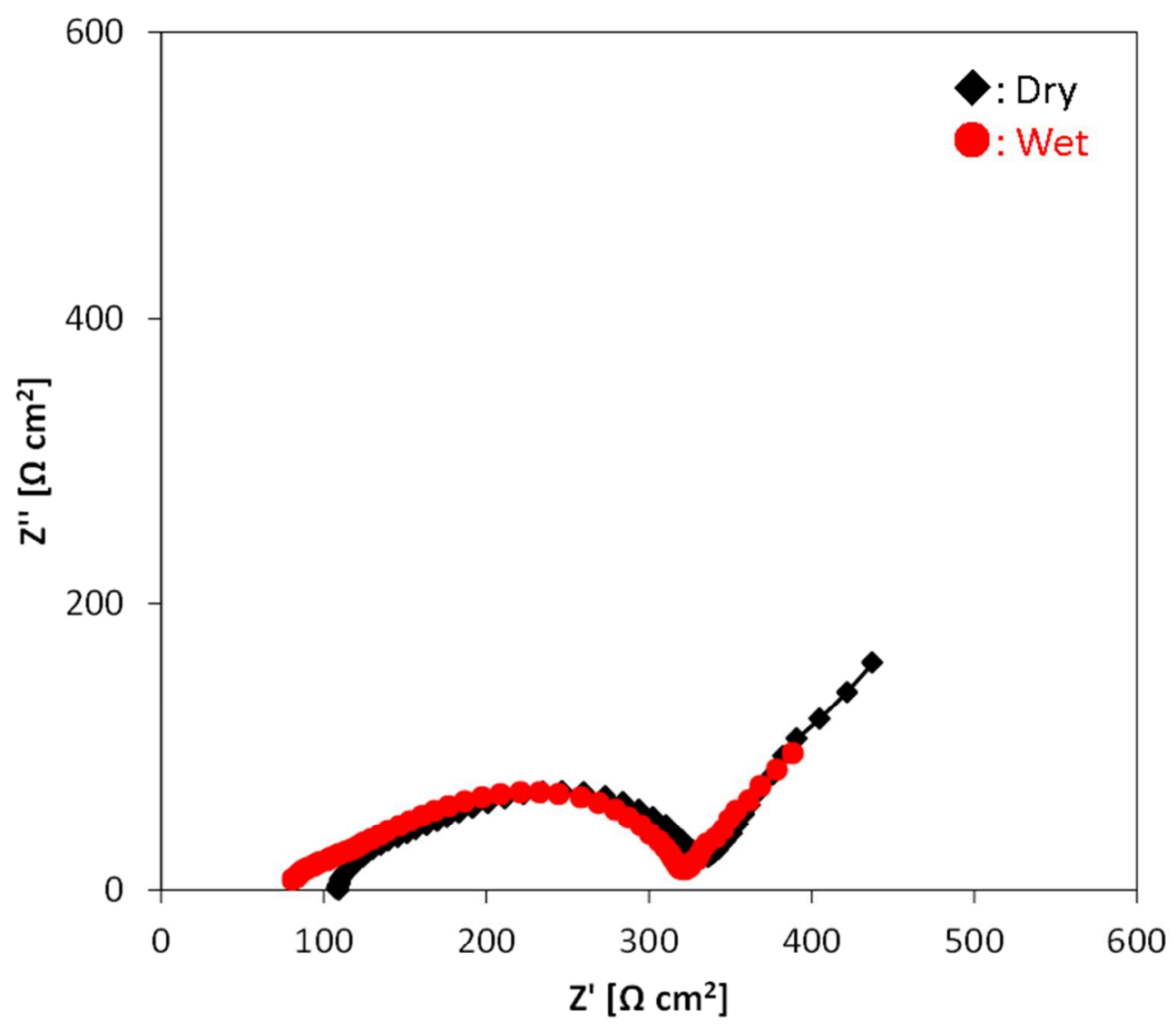

Figure S2. 


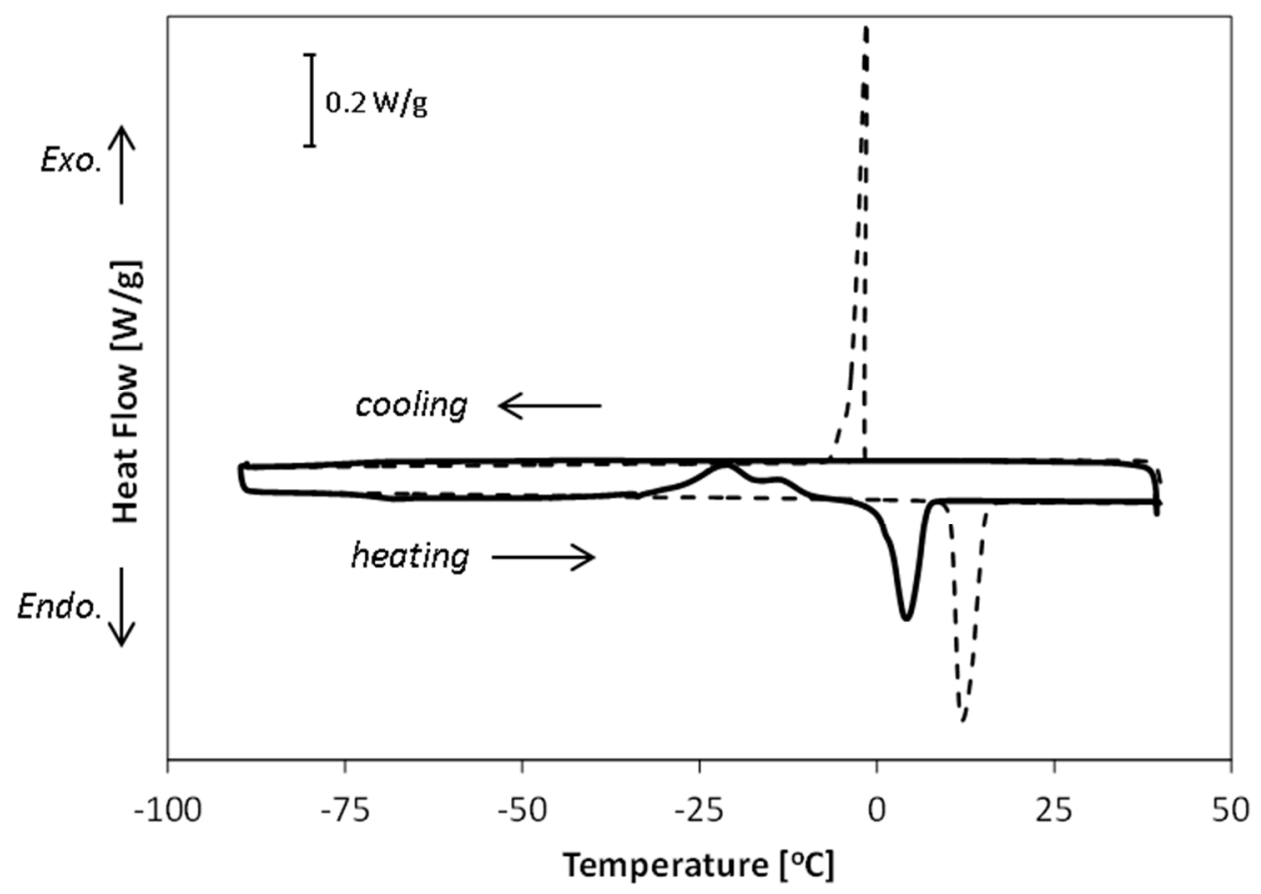

Figure S3. 